$39 \%)$ or a partner with an STD (27\% vs. $8 \%, p<0.0001)$, and less likely to be diagnosed with chlamydia ( $55 \%$ vs. $61 \%, p=0.007$ ). STD clinic patients reported having more partners (mean 16.3 vs. 11.7, $\mathrm{p}=0.0008$ ) in the prior year, and more methamphetamine $(18 \%$ vs. $11 \%, p<0.0001)$, Viagra (18\% vs. $13 \%, p=0.006)$ and popper use $(26 \%$ vs. $19 \%, p=0.0004)$. HIV-uninfected STD clinic patients were more often HIV-tested ( $84 \%$ vs. $78 \%, p=0.001$ ) at the time of STI diagnosis. HIV-infected MSM diagnosed in the STD clinic were less likely to have a primary HIV care provider than other MSM ( $80 \%$ vs. $96 \%$, p < 0.0001).

Conclusion STD clinics serve an economically disadvantaged, high risk and disproportionately symptomatic subset of the larger population of MSM with bacterial STI.

\section{P3.145 SCREENING FOR ANAL DYSPLASIA IN HIV POSITIVE AND HIV NEGATIVE MEN WHO HAVE SEX WITH MEN USING ANAL CYTOLOGY AND P16/INK4 IMMUNOSTAINING; A CROSSECTIONAL STUDY}

doi:10.1136/sextrans-2013-051184.0604

R Arora, D Pandhi, K Mishra, S N Bhattacharya, V A Yhome. University College Of Medical Sciences \& Guru Teg Bahadur Hospital, New Delhi, India

Background With legal acceptance of homosexuality in India, men who have sex with men (MSM) are increasingly being reported. Akin to cervical cancer in sexually active women, MSM are predisposed to anal cancers especially with co-infection of HIV. Further, screening of Papanicolaou stained anal smears in MSM can greatly reduce morbidity and mortality due to anal cancer; similar to cervical Pap screening in sexually active women. P16/ink4, a surrogate marker for oncogenic HPV coinfection, improves diagnostic accuracy for anal dysplasia. However, there are only two studies from Asia and none from India analysing anal dysplasia in MSM. This cross-sectional study endeavoured to assess the prevalence of anal dysplasia using Pap smears and p16 immunostaining amongst MSM in India.

Methods A total of 31 consecutive HIV positive and 34 HIV negative MSM ( $\mathrm{n}=65$ ) were subjected to anal cytological evaluation with Pap stain and p16 staining. Chi-square test and coefficient of correlation were used for comparison.

Results Twenty four (36.9\%) and thirty one (47.6\%) patients were transgender and bisexual respectively. Eighteen (27.7\%) had abnormal anal cytology which was higher in HIV positive as compared to HIV negative group ( $35 \%$ versus $20 \%, p=0.180$ ). Similarly, both low grade $(25.8 \%$ vs $17.6 \%, p=0.549)$ and high grade lesions (8.3\% vs $4.8 \%, p=0.341$ ) were comparable in HIV positive and negative groups. A negative correlation of CD4

\section{Abstract P3.145 Table 1}

\begin{tabular}{llll}
\hline \multicolumn{4}{l}{ Anal cytology results $(\mathbf{H I V})$} \\
\hline Anal cytology & Overall $(\mathbf{n}=\mathbf{6 5})$ & HIV positive $(\mathbf{n 1}=\mathbf{3 1})$ & HIV negative $(\mathbf{n} \mathbf{2}=\mathbf{3 4})$ \\
\hline Low grade dysplasia & $14(21.5 \%)$ & $8(25.8 \%)$ & $6(17.6 \%)$ \\
High grade dysplasia & $4(6.15 \%)$ & $3(9.6 \%)$ & $1(2.9 \%)$ \\
Total & $18(27.7 \%)$ & $11(35.5 \%)$ & $7(20.6 \%) \mathrm{p}=0.180$ \\
\hline
\end{tabular}

\section{Abstract P3.145 Table 2}

\begin{tabular}{lll}
\hline Anal cytology results (Transgender) & \\
\hline Cytology & Transgender $(\mathbf{n 3}=\mathbf{2 4})$ & Non-transgender $(\mathbf{n 4}=\mathbf{4 1})$ \\
\hline Low grade dysplasia & $8(33.3 \%)$ & $6(14.6)$ \\
High grade dysplasia & $2(8.3 \%)$ & $2(4.8 \%)$ \\
total & $10(41.7 \%)$ & $8(19.5 \%) p=0.08$ \\
\hline
\end{tabular}

count at time of recruitment with degree of anal dysplasia was observed $(r=-0.073, p=0.696)$. A total of $13(20 \%)$ smears were P16 positive with sensitivity, specificity, positive predictive value and negative predictive value of $72.3 \%, 100 \%, 100 \%$ and $92.3 \%$ respectively.

Conclusion Anal cytology should be used to screen for anal dysplasia in MSM irrespective of HIV status. Furthermore, addition of P16, with greater specificity for high grade lesions, will significantly improve diagnostic accuracy.

\section{P3.146 RISING STI PREVALENCE AMONG MSM CLIENTS IN CALABAR, NIGERIA: A CALL FOR ACTION}

doi:10.1136/sextrans-2013-051184.0605

K M Ugoh. Initiative for Improved Male Health, Calabar., Nigeria

Introduction Evidence has shown that there are increasing rates of new HIV and other STI infections occurring among Men who have Sex with Men (MSM) in Nigeria, with the prevalence 3 times higher than the general population as reported by the 2011 National Integrated Bio Behavioral Surveillance Survey. The poor state of health care and support services hinders our effort to control the high rates of these new infections among MSM.

Methods The Initiative for Improved Male Health (IMH-Initiative) works to provide a safe space for young MSM living with HIV to access comprehensive palliative care and support, as well as referrals for other services through drama and dance competitions. An STI assessment was conducted in IMH-Initiative's Community Center in Calabar, for gay men and other MSM. An STI history was conducted for all clients who visited the community clinic specifically for HCT and STI counselling and referrals within a 5 month period, and their data were collated.

Results 61 MSM were diagnosed, and reported the following in the last 6 months. 49 where living with HIV. 46 had previous histories of untreated anal warts. 20 had previous histories of treated Gonorrhea by self medication and herbs. 21 had untreated boils and rashes around the genitals. 10 clients where living with HIV, and reported untreated penile and rectal gonorrhoea. All clients indicated that there were not comfortable discussing STI infections with staff of public hospitals.

Conclusion It is evident that a reasonable number of STI infections among MSM are not completely treated. This thereby increases the individual's risk of HIV infection, and cripples HIV prevention programming in Nigeria. HIV programmes targeting MSM must incorporate STI syndromic management, so as to increase access to non-stigmatised diagnosis and treatment of STIs. Also, access to STI drugs for clients cannot be overemphasised.

\section{P3.147 PREVALENCE OF HIV/STIS AND RISK BEHAVIOR IN MEN WHO HAVE A SEX WITH MEN, BELIZE, 2012}

doi:10.1136/sextrans-2013-051184.0606

'S Morales-Miranda, 'B Alvarez, ${ }^{2} \mathrm{M}$ Manzanero. 'Universidad del Valle de Guatemala, Guatemala, ${ }^{2}$ Ministry of Public Health Belize, Belize

Background In Central America, the HIV epidemic is primarily concentrated in a few populations; including men who have a sex with men (MSM). The lack of reliable HIV prevalence estimates among MSM has impacted HIV programme planning targeting this population. In 2012, we conducted the first biological and behavioural survey among MSM in Belize.

Methods From January - July 2012, we used respondent-driven sampling to recruit MSM in four districts of Belize. Data was collected using a behavioural survey questionnaire administered through audio computer-assisted self-interview (ACASI). All participants were tested for HIV, syphilis and herpes simplex virus 